\title{
Primary non-Hodgkin's lymphoma of the mandible presenting following tooth extraction
}

\author{
S. J. Parrington, ${ }^{1}$ and A. Punnia-Moorthy, ${ }^{2}$
}

\author{
A case of oral non-Hodgkin's lymphoma presenting following \\ a tooth extraction is reported. The clinical presentation was \\ that of a periodontal lesion and an extraction socket that \\ failed to heal, which may have led to initial confusion in \\ diagnosis and management.
}

Malignant lymphomas are a diverse group of neoplasms affecting the lymphoreticular system. Hodgkin's disease is a condition characterised histologically by the presence of large multi-nucleated ReedSternberg cells. All other neoplasms of the lymphoid system are referred to as nonHodgkin's lymphoma (NHL), and are derived predominantly from the cells of the B lymphocyte series. Hodgkin's disease and NHL commonly present as non- tender, enlarged lymph nodes, accompanied by diffuse symptoms of fatigue and lowgrade intermittent fever. The cervical, axillary and inguinal lymph node groups are typically affected. In contrast to Hodgkin's disease, NHL may develop extra-nodally outside the lymphoid system and can occur in the stomach, skin, lung, salivary glands and rarely in the mouth. ${ }^{1}$

Oral lesions of NHL may develop in the soft tissues or centrally within the jaws, frequently as one of many lesions throughout the body or rarely as a primary entity prior to spread to the regional lymph nodes. Oral lesions appear as nontender swellings commonly affecting the vestibule, gingiva or posterior hard palate and develop slowly, mimicking a dental abscess of endodontic or periodontal origin. $^{2}$ In contrast, a lesion arising in

${ }^{1}$ Lecturer, ${ }^{2}$ Senior Lecturer, Department of Oral Surgery, University of Sydney, Faculty Building 2, Chalmers Street, Surry Hills, Sydney NSW 2010, Australia

REFEREED PAPER

Received 16.10.98; accepted 11.02.99

(C) British Dental Journal 1999; 187: 468-470 bone may present with a vague pain or discomfort which might be mistaken for toothache.

Adults over the age of 60 years are most commonly affected by NHL, although children may be affected by more aggressive intermediate- and high-grade lesions. Almost all subtypes of NHL, including those with oral lesions, show a slight to moderate male preponderance. Treatment normally consists of radiotherapy with or without chemotherapy depending on staging of the disease. Outcome is largely determined by histological type and extent of the disease.

\section{Case report}

A 57-year-old, apparently healthy male Caucasian of Mediterranean origin, a cabinet-maker by profession, presented to his general dental practitioner complaining of a loose tooth and bleeding gums. On examination the gingiva around the lower right second premolar appeared grossly inflamed and the tooth exhibited grade II mobility. A periapical radiograph revealed an area of poorly defined radiolucency and diffuse alveolar bone loss extending distally from 5 (45), toward $\overline{8}$ (48) (Fig. 1). $\overline{5}$ was extracted and the patient advised to return 10 days later for review of the healing socket. At the review appointment the mucosal lesion had increased in size and was now protruding from the extraction site. The patient was promptly referred for the assessment and management of the enlarging soft tissue lesion.

At presentation in the specialist clinic the patient's medical and social histories were unremarkable, he neither smoked nor drank and had no known risk factors for HIV. On physical examination he was afebrile and there were no enlarged lymph nodes in the neck. Intra-oral examination revealed a large exophytic mass about $3 \mathrm{~cm} \times 3 \mathrm{~cm}$ in size arising from the extraction socket of 5 (Fig. 2). A provisional diagnosis of pyogenic granuloma was made and an incisional biopsy was performed.

Microscopic examination of the sections showed squamous mucosa with a dense subepithelial infiltrate of malignant cells extending to the deep margins of the biopsy (Fig. 3). Immunohistochemistry demonstrated positive CD marker

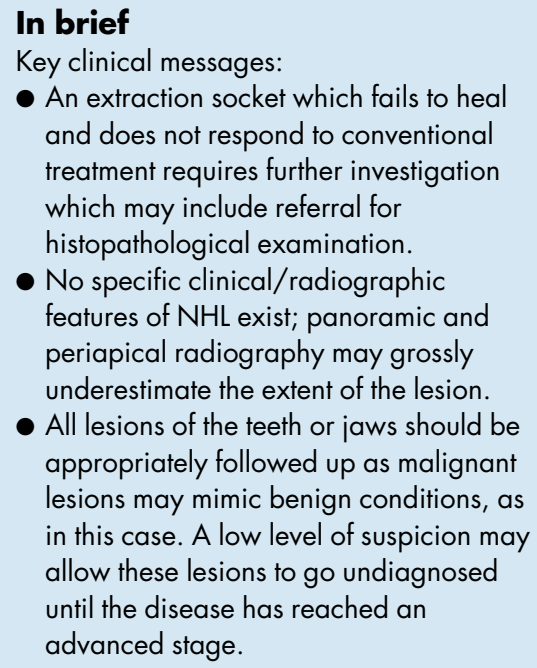
and does not respond to conventional treatment requires further investigation which may include referral for histopathological examination.

- No specific clinical/radiographic features of NHL exist; panoramic and periapical radiography may grossly underestimate the extent of the lesion.

- All lesions of the teeth or jaws should be appropriately followed up as malignant lesions may mimic benign conditions, as in this case. A low level of suspicion may allow these lesions to go undiagnosed until the disease has reached an advanced stage.

expression for B-cell markers CD 20 and CD 79a (Figs. 4a, b), negative results were obtained for T-cell markers CD3 and CD30. Based on the working formulation for classification of non-Hodgkin's lymphomas a diagnosis of an intermediategrade diffuse large B-cell malignant lymphoma was made. During staging of the disease which consisted of a physical examination, complete blood count, bone marrow biopsy and CT scans of the 


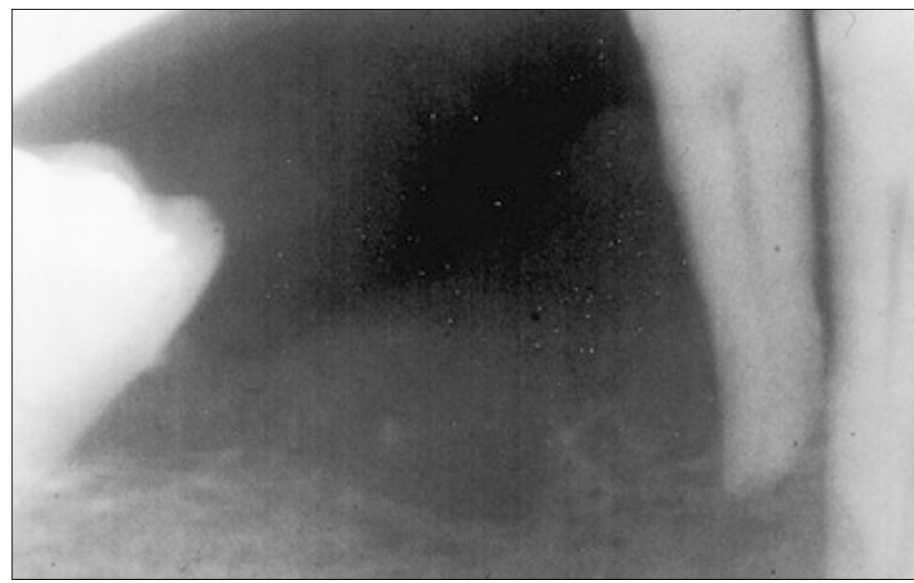

Fig. 1 Periapical radiograph at initial

presentation

showing diffuse

bone loss and ill-

defined

radiolucency

extending distally

from $51(45)$, to 8

(48)

head and neck, pelvic and abdominal regions, no other evidence of disease was present apart from the localised area of bone destruction in the body of the right mandible (Fig. 5). A diagnosis of primary non-Hodgkin's lymphoma of the mandible was made, and the patient underwent a course of radiotherapy to the mandible and neck, a total dose of 40 Gray over 4 weeks divided into 20 fractions. The immediate outcome was the complete resolution of the oral lesion with temporary side effects of hair loss and mouth ulcers.

\section{Discussion}

In most countries between 25 and 35\% of NHL cases occur extra-nodally and in 3\% of these cases the initial presentation may be in the oral cavity. Around 100 cases of mandibular NHL have been described in the literature. ${ }^{3}$ Although oral lesions of NHL are often a component of more widely disseminated disease, at times, as in this case, the lymphoma presents in the oral cavity as the first identifiable evidence of the disease. The prevalence of NHL is increasing among those who are immunocompromised, receiving organ transplants, and those with autoimmune disorders such as Sjögrens syndrome or rheumatoid arthritis. An increased inci-

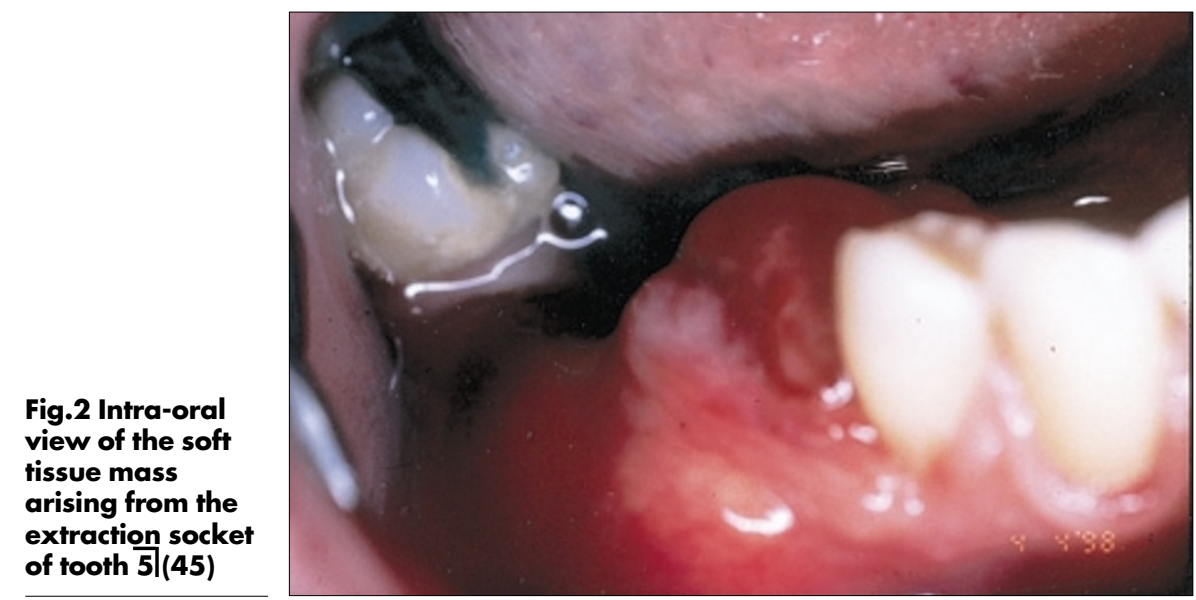

Fig.3

Photomicrograph showing

squamous mucosa and a dense subepithelial infiltrate of malignant cells, having vesicular nuclei and frequent mitoses. (H\&E stain, $\times 125$ )
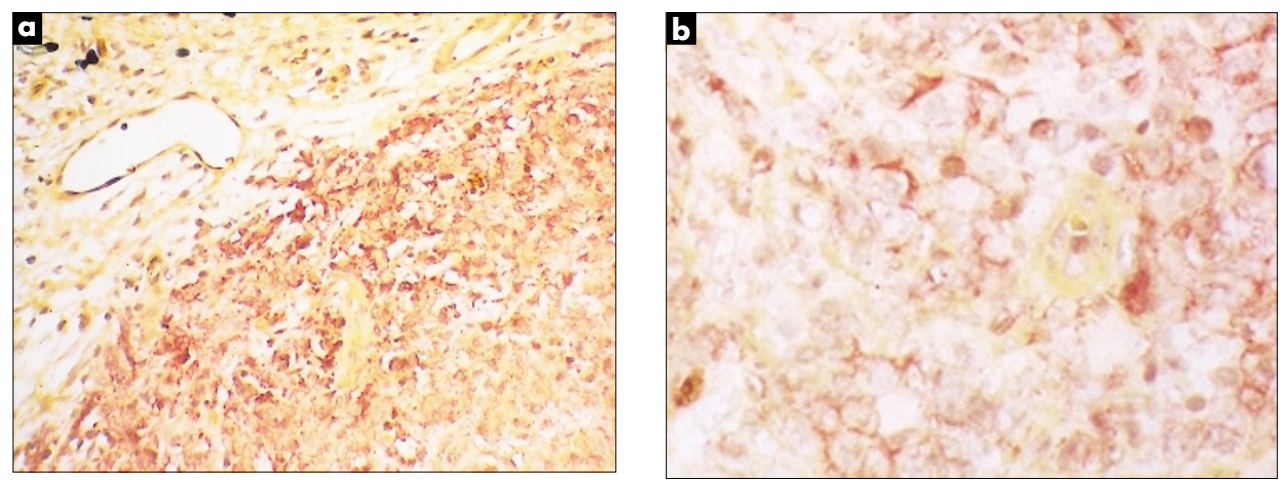

Fig.4 Photomicrograph showing positive B cell lymphoid marker expression. (a)CD20 and (b) CD 79a 


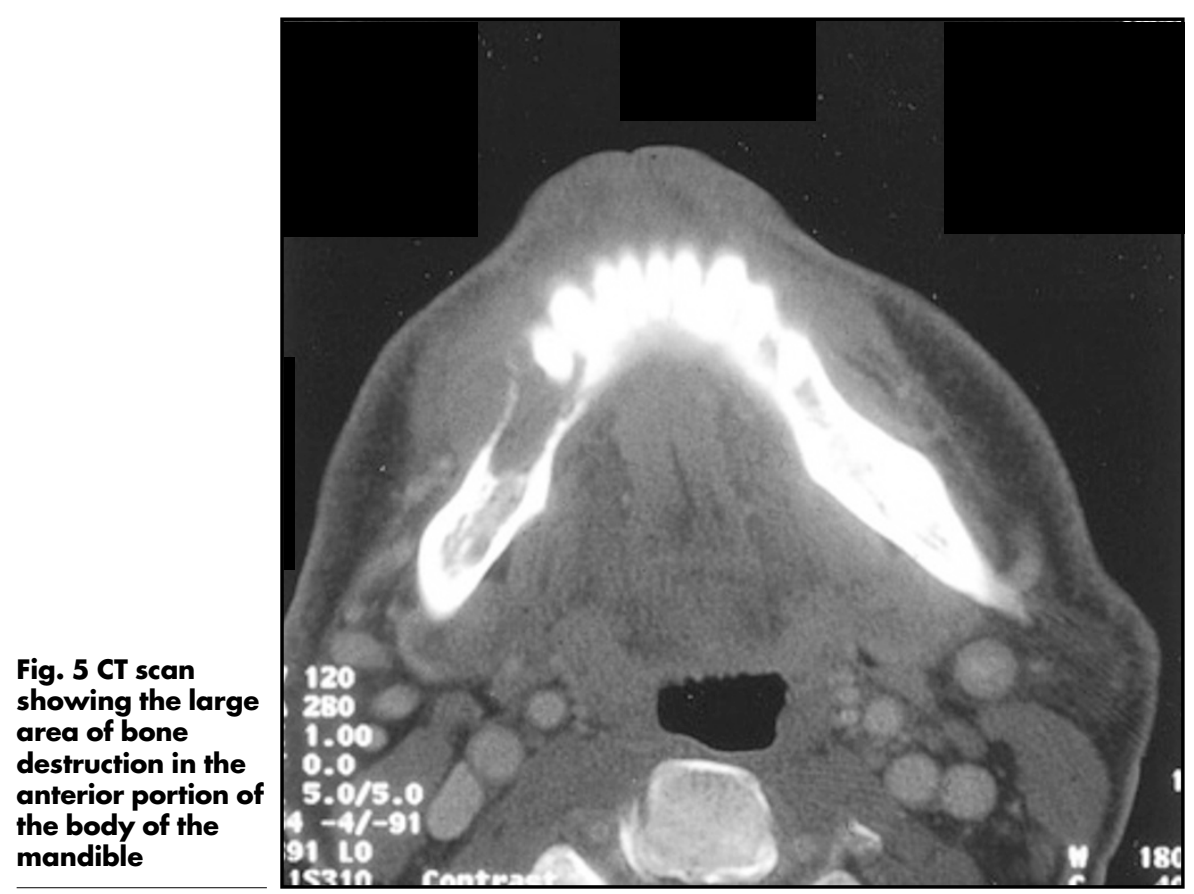

dence of NHL has also been recognised in patients suffering from AIDS, ${ }^{4}$ and oral lesions of NHL have presented as the first manifestation of AIDS. ${ }^{5}$ The increased incidence of NHL in the general population, however, remains unexplained. Studies originating from parts of northern Italy have suggested that there is a strong link between chronic infection with hepatitis $\mathrm{C}$ and an increased incidence of NHL. ${ }^{6}$ Recipients of blood and blood products also appear to be at increased risk of developing malignant lymphomas - whether this is caused by the transmission of viral pathogens is unknown.
There is considerable evidence that lymphomas at specific sites are preceded by the presence of a local inflammatory process. It is speculated that inflammation increases the rate of cell division of lymphocytes, thereby increasing the chance of a malignant clone developing. The clinical features of oral lesions of NHL, and the radiographic picture of an ill-defined or ragged radiolucency with loss of alveolar bone support, as in this case, may lead to confusion in diagnosis with infective processes such as osteomyelitis and other malignant conditions, for example squamous cell carcinoma or salivary gland tumours. Thus the

importance of initial histological examination should not be underestimated. ${ }^{1}$ Histological diagnosis can be difficult for the pathologist and it is important to handle the biopsy specimen carefully to avoid pressure artefacts.

Oral lymphoma can spread by three routes:

- Lymphatic

- Contiguous spread to adjacent structures

- Blood borne distant metastases in the later stages of the disease.

Primary NHL of the mandible for the most part is intermediate or highly malignant in nature but it tends to remain localised. ${ }^{3}$ Reports on survival rates vary widely, ranging from 11 to 38 months; however, Baccahud et al ${ }^{3}$ reported $60 \%$ of the patients in their series were alive and disease free after 5 years. The broad range of survival rates can be explained by the different degree of organ involvement in the patients studied, and by the varied histologic grades of malignancy and treatment regimes employed.

Extra-nodal NHL of the oral cavity is a rare finding, however, patients with oral lesions of NHL commonly present at the dental clinic in the first instance. Any delay in diagnosis has important implications on the morbidity and mortality of the condition.

The authors wish to thank: Professor D. E. Joshua, Institute of Haemotology; Dr C. Mcleod, Department of Radiation Oncology; and DrS. W. McCarthy, Department of Anatomical Pathology, Royal Prince Alfred Hospital, Camperdown, Sydney for their assistance in the preparation of this case report.

\section{Submitting illustrations to the BDJ}

Authors submitting manuscripts for publication in the BDJ are reminded that two copies of all illustrations must be supplied. This will assist in speeding up the refereeing process - manuscripts and illustrations are always sent to referees and a set of illustrations is required at the editorial office for reference. If you choose to submit 35-mm transparencies then one set of these and one set of prints (colour or black \& white) will be acceptable.

We strongly advise authors to keep copies of all illustrations submitted in case letters are lost or damaged in transit. Please do not send glass-mounted transparencies - even when securely wrapped the glass will often arrive cracked or shattered, resulting in damage to the surface of the slide which usually renders it unsuitable for reproduction. 